\section{Membranes for Specific Adsorption: Immobilizing Molecularly Imprinted Polymer Microspheres using Electrospun Nanofibers}

Roman Büttiker, Jürgen Ebert, Christian Hinderling, and Christian Adlhart*

${ }^{*}$ Correspondence: Dr. C. Adlhart, Zurich University of Applied Sciences ZHAW, Institute of Chemistry and Biological Chemistry, Einsiedlerstrasse 31, 8820 Wädenswil Tel.: +4158934 57 54, E-mail: christian.adlhart@zhaw.ch

Abstract: Molecularly imprinted polymer microspheres were immobilized within a polymer nanofiber membrane by electrospinning. Such membranes simplify the handling of functional microspheres and provide specific recognition capabilities for solid-phase extraction and filtration applications. In this study, microspheres were prepared by precipitation polymerization of methacrylic acid and divinylbenzene as a cross-linker with the target molecule (-)-cinchonidine and then, they were electrospun into a non-woven polyacrylonitrile nanofiber membrane. The composite membrane showed specific affinity for (-)-cinchonidine which was attributed to the functional microspheres as confirmed by Raman microscopy. The target molecule capturing capacity of the composite membrane was $5 \mathrm{mg} / \mathrm{g}$ or $25 \mathrm{mg} / \mathrm{g}$ immobilized functional microsphere. No difference in target affinity was observed between the immobilized microspheres and the free microspheres. These results reveal that electrospun composite membranes are a feasible approach to immobilizing functional microspheres.

Keywords: Electrospinning · Filtration - Molecularly imprinted polymer · Precipitation polymerization · Raman microscopy

\section{Introduction}

Easy-to-use membranes for solid-phase extraction (SPE) offering specific recognition are suitable for numerous energyefficient separation applications from the selective removal of estrogenic substances in waste water to the chiral separation of pharmaceutical products. ${ }^{[1,2]}$ The separation efficiency of a membrane depends mainly on the specific affinity between the membrane and the target molecule, i.e. its molecular recognition, and the diffusion of the substrate into the membrane - a factor that is mainly controlled through the membrane's surface and porosity. ${ }^{[3]}$

Synthetic polymers with tailor-made molecular recognition abilities, so called molecularly imprinted polymers (MIPs) seem particularly suited in controlling the specific affinity for the target molecules. ${ }^{[4]}$ These MIPs are similar to specific biological receptors, such as enzymes or antibodies, but they have a greater chemical and physical stability and they can be manufactured on a large scale and in a simple manner.

Increasing the MIPs surface is achieved by reducing the particle size and recently, numerous methods such as precipitation polymerization were established to produce MIP micro- or nanospheres. ${ }^{[5,6]}$ Typically, these micro- or nanospheres are packed into columns to be used as solid-phase extraction (SPE) material for analytical purposes.
For large-scale applications in filter membranes, immobilizing the MIP microspheres becomes mandatory. A promising technique is their immobilization in membranes of electrospun nanofibers, as these membranes firstly have a very large porosity required for an efficient access of the filtrate and secondly because the smaller nanofibers may not cover the entire MIP surface of the larger microspheres thereby restricting the access of the substrate to the functional MIPs' surface. ${ }^{[7-9]}$ An alternative approach to the immobilization of MIP microspheres in nanofiber membranes is the direct imprinting of the membrane's nanofibers during the electrospinning process ${ }^{[3]}$ or the chemical postmodification of the nanofiber's surface using grafting techniques. ${ }^{[10]}$ An essential question for immobilized MIP microspheres is in how far their molecular recognition properties are changed within the membrane's matrix.

There is a large interest in developing MIPs with specific recognition abilities for propranolol and atenolol - two $\beta$-blocking agents - which are together with cinchonidine (Fig. 1), an alkaloid having antimalaria activity - well-documented model compounds for molecular imprinting. ${ }^{[6,11]}$

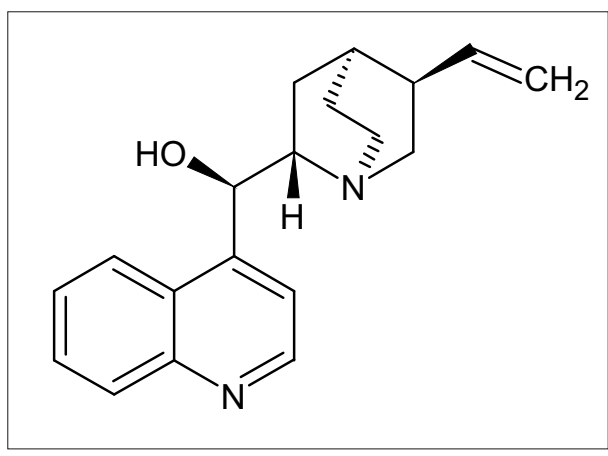

Fig. 1. Structure of the template molecule (-)-cinchonidine

In this work, we synthesized by precipitation copolymerization of methacrylic acid (MAA) and divinylbenzene (DVB) MIP microspheres with 4-5 $\mu \mathrm{m}$ in diameter for the molecular recognition of cinchonidine. The microspheres were immobilized by incorporating them into membranes of polyacrylonitrile (PAN) nanofibers with different diameter through electrospinning. The molecular recognition capacity of the free microspheres as well as the ones incorporated within the PAN membrane matrix was quantified by guest-binding experiments and the results were compared with the corresponding non-imprinted polymers (NIPs), where no template molecules had been added during the precipitation polymerization.

The composite PAN/MIP membranes were characterized using scanning electron microscopy (SEM) and Fourier transform infrared spectroscopy (FT-IR). We were also able to visualize the location and distribution of the bound target molecules within the MIP membranes by Raman microscopy.

\section{Experimental}

All chemicals were purchased from Sigma-Aldrich and applied without further purification unless otherwise mentioned. 


\section{Synthesis of MIP Microspheres Imprinted with (-)-Cinchonidine}

MAA/DVB-MIP and MAA/DVB-NIP microspheres were synthesized following the procedure of Liu et al. ${ }^{[6]}$ MIPs were obtained by dissolving $114.1 \mathrm{mg}(-)$-cinchonidine (the template; $0.388 \mathrm{mmol}$ ), $144.4 \mathrm{mg}$ methacrylic acid (MAA; $1.70 \mathrm{mmol}$ ), $948.3 \mathrm{mg}$ divinylbenzene (DVB; $7.28 \mathrm{mmol}$ ) and $77.9 \mathrm{mg}$ azobisisobutyronitrile (AIBN; $0.47 \mu \mathrm{mol}$ ) in $32 \mathrm{ml}$ acetonitrile/toluene $(3: 1 \mathrm{v} / \mathrm{v})$ in a $50 \mathrm{ml}$ Schott flask. Degassing was achieved by bubbling argon through the solution for 5 minutes and keeping the Schott flask under argon while closing. The flask was placed into a glass vessel filled with sand to fix the flask and the vessel was connected with a Büchi R-210 rotary evaporator to rotate the vessel at $20 \mathrm{rpm}$ for $18 \mathrm{~h}$ while the oil bath temperature was continuously augmented from $25^{\circ} \mathrm{C}$ to $70{ }^{\circ} \mathrm{C}$ within the first 2 $\mathrm{h}$ and then kept constant. The generated polymer was separated by sedimentation and then extracted with $200 \mathrm{ml}$ methanol and allowed to sediment for $12 \mathrm{~h}$. The supernatant was decanted off and the extraction step was repeated two times with methanol, once with water, and two times with tetrahydrofurane. Finally, the 4-5 $\mu \mathrm{m}$ MIPs microspheres were dried in air at ambient temperature. The corresponding non-imprinted microspheres (NIPs) were synthesized accordingly but without adding the template.

\section{Electrospinning}

The electrospinning apparatus followed the set-up of Doshi and Reneker having a horizontal spraying direction. ${ }^{[12]} 5 \mathrm{ml}$ of sample solution (polyacrylonitrile, $10 \%$ in dimethylformamide, w/v; $2.5 \%$ MIPs/NIPs - giving a ratio of $4: 1$ between PAN and MIPs/NIPs for the composite fabric; components were dispersed by ultrasonication) were pumped at $8 \mu \mathrm{l} / \mathrm{min}$ through an insulating Teflon tubing (i.d. $0.60 \mathrm{~mm}$ ) to the steel spraying capillary $(0.90 \times 70 \mathrm{~mm})$ using a syringe pump (Lambda VIT-FIT). The capillary was kept at $+15 \mathrm{kV}$ using a high-voltage power supply (fug HCN 35-35000). The planar aluminium collector (30 $\times 30 \mathrm{~cm}$ ) was placed at a distance of $10 \mathrm{~cm}$ and set on ground. The collected fabric was then dried for a day on air at ambient temperature.

\section{Scanning Electron Microscopy (SEM)}

The SEM image was acquired on a FEI quanta FEG scanning electron microscope equipped with a back-scattered electron detector by Intertek Expert Services (see Acknowledgement). The sample was coated with a thin layer of platinum by physical vapor deposition.

\section{FT-IR Spectroscopy}

Diamond attenuated total reflectance (D-ATR) FT-IR spectra at different positions of the electrospun composite nanofiber membranes as well as from their component materials were recorded on a Bruker Tensor 37 FTIR spectrometer at $4 \mathrm{~cm}^{-1}, 64$ scans using the Specac Golden Gate ATR accessory confirming the presence of the MIP and NIP microspheres within the membranes. The spectra of the membranes also showed the presence of additional DMF, the solvent used during electrospinning.

\section{Raman Microscopy and Imaging}

Raman spectra $\left(0-3800 \mathrm{~cm}^{-1}\right)$ of the electrospun membranes were recorded by scanning an area of $40 \times 40 \mu \mathrm{m}^{2}$ with a lateral resolution of $0.27 \mu \mathrm{m}$ thus acquiring a total of 22500 spectra. Data were collected with a Witec alpha300 Raman microscope equipped with a 1024 CCD detector (Witec, Ulm, Germany) through a Nikon CF Plan 100x 0.95 NA EPI objective using a frequency doubled Nd:YAG laser for excitation at $532 \mathrm{~nm}$. The average accumulation time for each spectrum was about $2 \mathrm{~s}$. Color-coded Raman images were generated from the scores of direct classical least square (DCLS) fits of the recorded Raman spectra (spectral range was 3140-2800, 2320-2180 and 1720-480 $\mathrm{cm}^{-1}$ ) obtained by using appropriate component spectra of the target molecule, the MIPs and NIPs respectively, the PAN nanofiber, and by applying a third-order polynomial background correction using the built-in fitting routine of the instrument's control software.

\section{Guest-binding Experiments}

To test the recognition ability of the MIPs/NIPs with and without the membrane, samples were each loaded with solutions containing the target molecule $(114 \mathrm{mg} / \mathrm{l}(-)$-cinchonidine in acetonitrile) which were then analyzed chromatographically and spectroscopically. $20 \mathrm{mg}$ MIPs/NIPs were stirred in $5 \mathrm{ml}$ template solution at room temperature for $4 \mathrm{~h}$ and then centrifuged (4500 $\mathrm{rpm}, 10 \mathrm{~min})$. The template concentration in the supernatant of the sample was then determined by HPLC while the loaded MIPs/NIPs were characterized by confocal Raman microscopy. The same tests were carried out with the membranes. $100 \mathrm{mg}$ membrane (equivalent to approximately $80 \mathrm{mg}$ PAN and $20 \mathrm{mg}$ MIPs/NIPs) were loaded for $4 \mathrm{~h}$ at room temperature within $5 \mathrm{ml}$ template solution and separated by centrifugation.

\section{High-performance Liquid Chromatography (HPLC)}

HPLC-only measurements were performed on an Agilent 1200 Series with multi-wavelength detector (MWD). Column: Modulo-Cart QC Strategy 5 C18-2 at $40{ }^{\circ} \mathrm{C}$; injection volume: $50 \mu \mathrm{l}$; eluent: water/methanol $(0-100 \%$ in $10 \mathrm{~min}$, then $100 \%$ methanol); flow rate $1 \mathrm{ml} / \mathrm{min}$; Detection at $234 \mathrm{~nm}$. peak area at retention time $7.6 \mathrm{~min}((-)$-cinchonidine $)$.

\section{Results}

\section{Morphology and Composition of the Affinity Membranes}

The electrospun PAN membranes were removed from the aluminium collector plate and a flexible self-supporting membrane material was obtained, which is easily handed at ambient atmosphere (Fig. 2). The SEM image provided the detailed morphology of the PAN/MIP membrane. The membrane is built by a non-woven network of nanofibers with a typical thickness of $200 \mathrm{~nm}$ - some fibers showing a thickness of up to $500 \mathrm{~nm}$ - and spherical particles of 4-5 $\mu \mathrm{m}$ are embedded within the fibers. Those spherical particles are clearly sitting like beads at the center of the fibers which can be regarded as the extension of the microspheres' axis. The non-woven nanofibers provide also a high porosity giving a membrane with a coherent network of voids. The chemical composition and the ratio of PAN and MIPs was evaluated by FT-IR spectroscopy.

D-ATR FT-IR spectra of membranes electrospun from 1:4 suspensions of MIP/NIP microspheres and PAN in DMF are given in Fig 3. When comparing those spectra with spectra of the free microspheres as well as of a PAN membrane without microspheres, it becomes evident that both components, i.e. microspheres and PAN nanofibers, are found within the electrospun membranes. For example the strongest peaks of PAN, the C-N stretching vibration at 2243 and the strong $\mathrm{CH}_{2}$-deformation vibration at $1453 \mathrm{~cm}^{-1}$ are observed in the composite PAN/MIP membrane as well as the indicative $\mathrm{CH}$ out-of-plane vibrations of the microspheres' polymer at $900,830,795$ and $708 \mathrm{~cm}^{-1}$. Further peaks at $1666,1388,1095$, and $661 \mathrm{~cm}^{-1}$ are attributed to DMF, which has not been completely removed from the membrane after the electrospinning process by drying the membrane on air for 24 $h$ at ambient temperature. D-ATR FT-IR spectra at different positions of the electrospun membranes show that the relative amount of microspheres and PAN nanofibers remains constant over the entire membrane surface. 


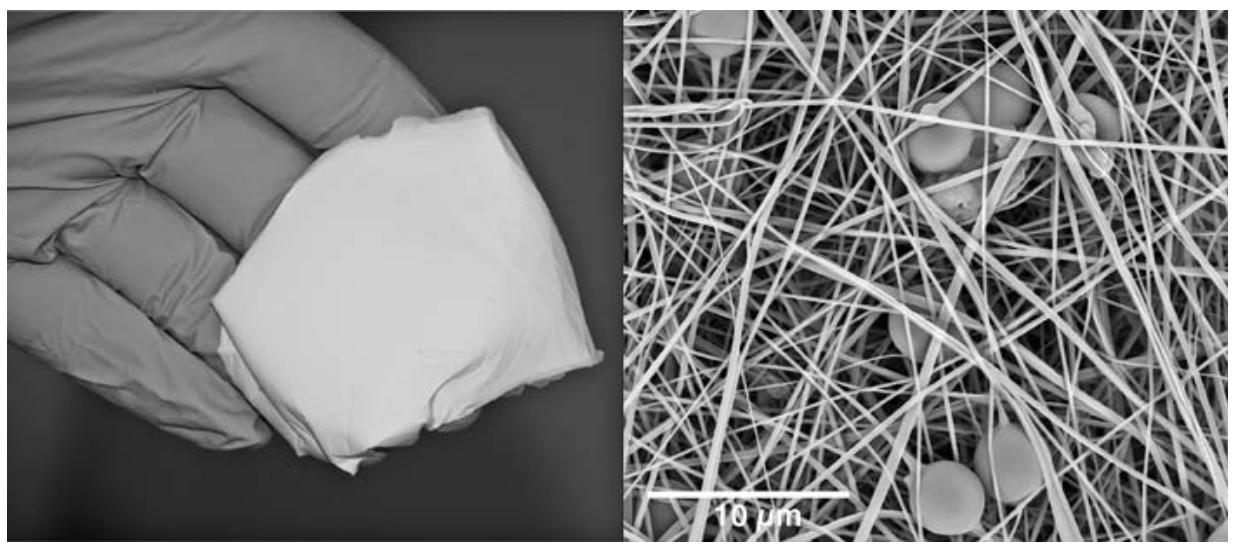

Fig. 2. Photography of an electrospun PAN nanofiber membrane and SEM image of the corresponding PAN/MIP affinity membrane.

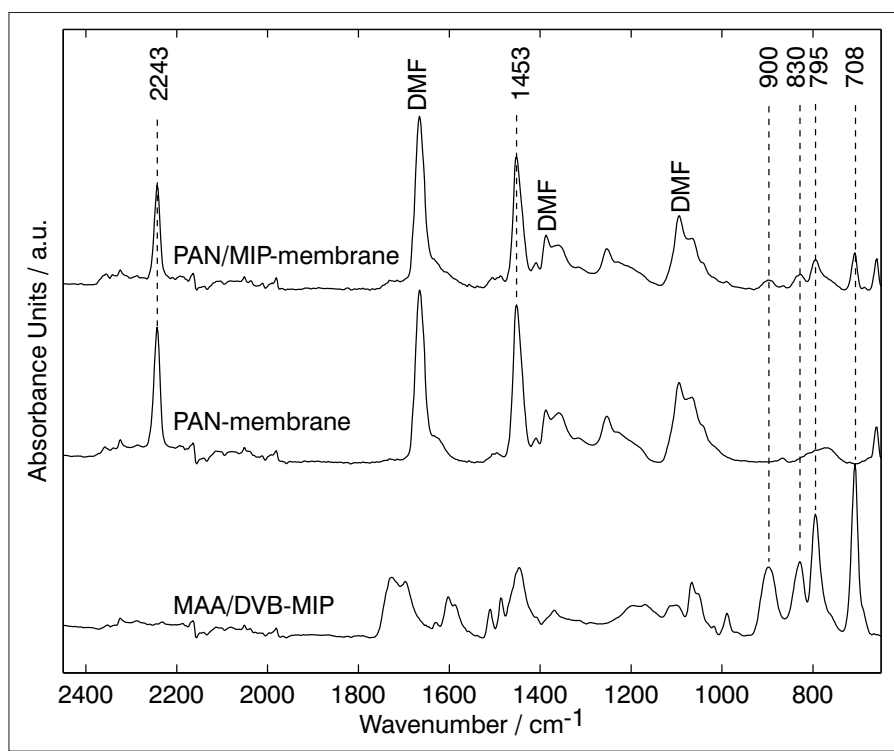

Fig. 3. D-ATR FT-IR spectra of the PAN/MIP membrane (top), the PAN membrane (middle), the and MIP microspheres (bottom).

\section{Direct Observation of Target Binding by Raman Spectroscopy}

Raman spectra of MAA/DVB-MIP microspheres immobilized within the PAN membrane were recorded before and after loading with the target molecule cinchonidine (Fig. 4). The major Raman bands are observed in the region of the aromatic $\mathrm{C}=\mathrm{C}$ stretching vibrations $\left(1618 \mathrm{~cm}^{-1}\right.$ and $\left.1638 \mathrm{~cm}^{-1}\right)$ as well as the in-plane $\mathrm{C}-\mathrm{H}$ deformation vibration $\left(1008 \mathrm{~cm}^{-1}\right)$ as expected for the MAA/DVB copolymer. The spectrum of a loaded MIP microsphere within the PAN membrane shows an additional smaller peak at $1368 \mathrm{~cm}^{-1}$ which is only present as a small shoulder in the unloaded MIP microsphere. This peak at $1368 \mathrm{~cm}^{-1}$ is in coincidence with the strongest Raman band of cinchonidine, a coupled $\mathrm{C}=\mathrm{N}, \mathrm{C}=\mathrm{C}$ stretching vibration of the quinoline backbone. The intensity of the $1368 \mathrm{~cm}^{-1}$ peak in the Raman spectrum of the loaded and immobilized MIPs indicates the amount of cinchonidine loading, since the Raman signals of the PAN matrix do not interfere with peaks in the $1360-1380 \mathrm{~cm}^{-1}$ region. The spectral difference between loaded and unloaded MIP was also exploited when studying the distribution of the target molecule within the composite PAN/MIP affinity membranes.

Raman images of the distribution of PAN and MIP microspheres within the composite membrane were obtained by mapping the membrane at $0.27 \mu \mathrm{m}$ steps and fitting the recorded Raman spectra with suited component spectra of PAN and MIP.

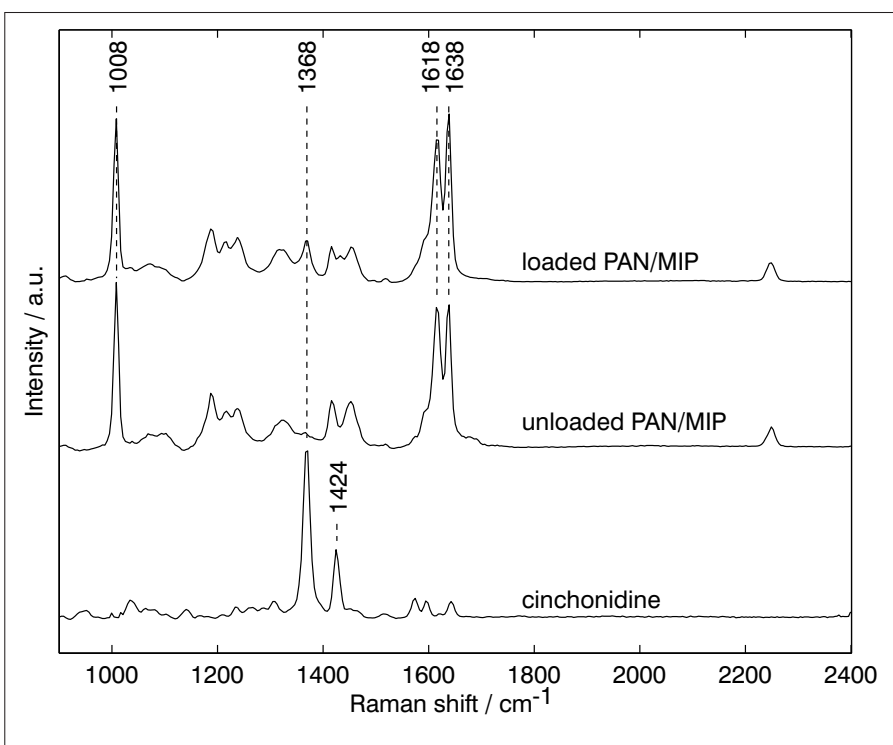

Fig. 4. Direct observation of target binding in a loaded MIP microsphere by a characteristic peak at $1368 \mathrm{~cm}^{-1}$ in the Raman spectra of a loaded (top) and an unloaded (middle) MAA/DVB-MIP electrospun into a PAN membrane. Bottom: Raman spectrum of the cinchonidine target molecule.

Two out of 22500 Raman spectra obtained by mapping are given in Fig. 5 for a MIP-rich and a PAN-rich region. The color-coded Raman image in the left hand side of Fig. 5 (bottom) reveals the chemical composition of the PAN/MIP membrane: a mesh of randomly oriented PAN nanofibers of $200-500 \mathrm{~nm}$ in diameter (blue) builds the support for the MIP microspheres with their diameter of approx. $5 \mu \mathrm{m}$ (green). Some MIP microspheres were below or above the focal plane of the Raman microscope and therefore appeared as smaller and diffuser spheres (top left corner). Whether the MIP microspheres were incorporated into the PAN nanofibers and covered with a thin PAN film or whether they were sitting on top of the fibers, remained unresolved. The Raman image on the right hand side of Fig. 5 (bottom) shows the distribution of PAN and the target molecule cinchonidine obtained from the identical Raman data as on the left hand side. Interestingly, cinchonidine perfectly collocates with the MIP microspheres. In addition, smaller cinchonidine-containing areas were found at the intersection points of the PAN nanofibers.

\section{Affinity Studies}

Guest-binding experiments were carried out to evaluate the affinity of the imprinted microspheres for the target molecules first before immobilizing them within the PAN membrane and second for the immobilized microspheres. Results for the cin- 


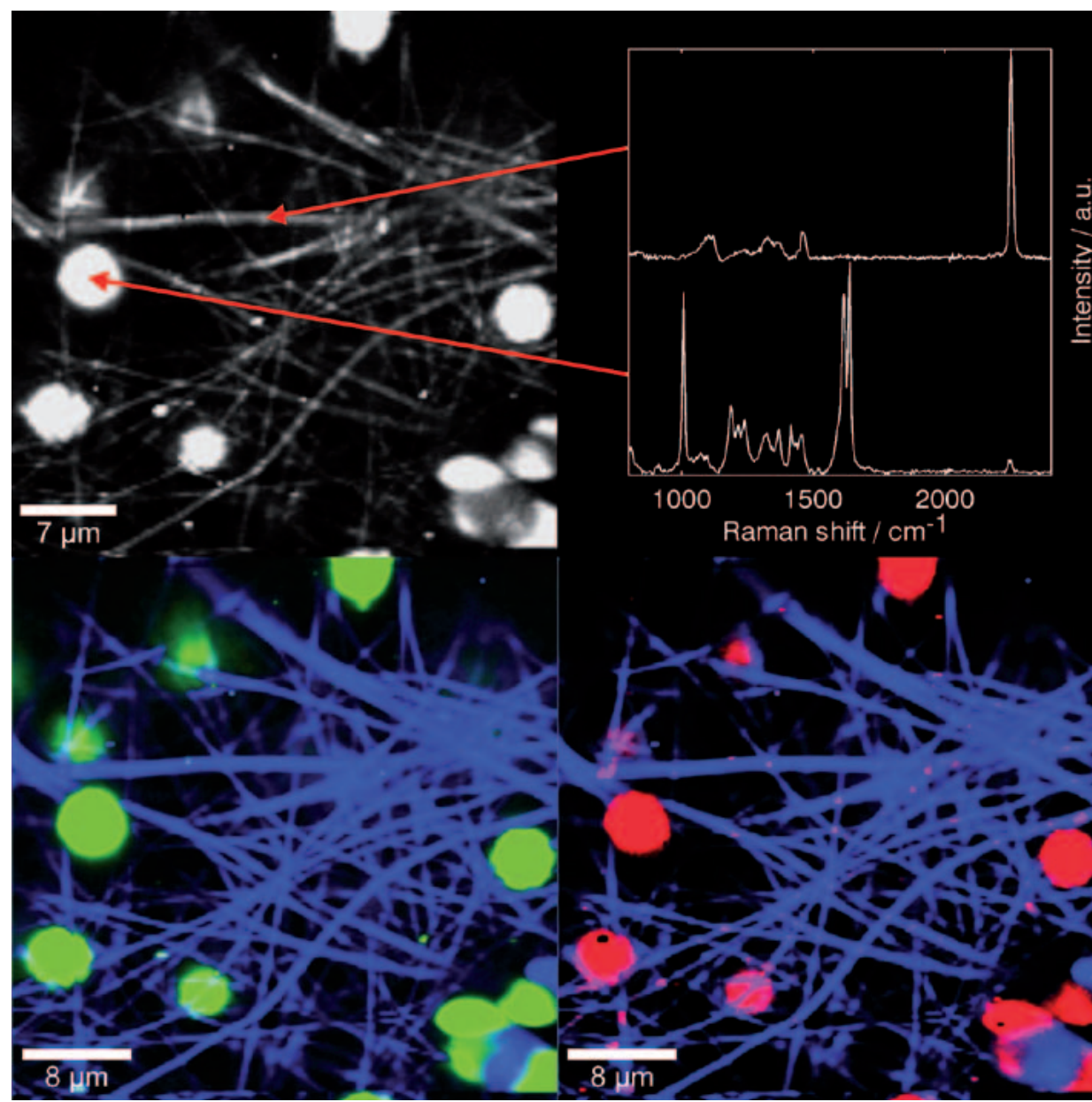

Fig. 5. Raman intensity map of a loaded PAN/ MIP membrane $\left(40 \times 40 \mu \mathrm{m}^{2}\right.$, top left) and individual Raman spectra at a PAN rich region (top right) and a MIP rich region (below). Bottom: Corresponding Raman images. The left hand image displays the distribution of the PAN nanofibers (blue) and the incorporated MIP microspheres with their diameter of $4-5$ $\mu \mathrm{m}$ (green). The right hand image displays the distribution of the target molecule cinchonidine (red).

chonidine-imprinted polymer are given in Table 1. Both MIP and NIP microspheres were found to reduce the concentration of the cinchonidine target molecule. The MIP microspheres reduced the concentration of cinchonidine in the acetonitrile solution by roughly $89 \%$ while the NIP microspheres reduced the concentration by roughly $59 \%$. When the same microspheres were immobilized in the PAN membrane, a reduction of $88 \%$ and $64 \%$ was observed, notably - within the experimental error - identical amounts between immobilized and free MIP and NIP microspheres. The PAN membrane alone did not adsorb significant amounts of cinchonidine (3\%).

Table 1. Relative concentration of the target molecule cinchonidine in the supernatant after guest-binding experiments of MIP/NIP microspheres alone and embedded into the PAN nanofiber membrane including the $95 \%$ confidence level. The concentration of the target solution was $114 \mathrm{mg} / \mathrm{l}$.

$\begin{array}{lll}\text { Sample } & \begin{array}{l}\text { free microspheres } \\ 100 \bullet c_{\text {rel }} \text { (cinchonidine) }\end{array} & \begin{array}{l}\text { immobilized microspheres } \\ 100 \bullet c_{\text {rel }}(\text { cinchonidine })\end{array} \\ \text { PAN } & - & 97.2 \pm 14.0 \\ \text { NIP } & 41.0 \pm 21.2 & 35.8 \pm 5.0 \\ \text { MIP } & 10.9 \pm 3.8 & 12.3 \pm 5.3\end{array}$

\section{Discussion}

Recently, several applications of nanofiber membranes for the specific adsorption of potentially harmful contaminants dissolved or dispersed in water have been reported. ${ }^{[1,2]}$ Their specific surface area of typically $10-80 \mathrm{~g} / \mathrm{m}^{2}$ and high porosities of often $80 \%$ and their interconnected network of voids facilitates the transfer of molecules and renders membranes of electrospun nanofibers well suited for filtration or solid-phase extraction applications. The different approaches for nanofiber membranes with recognition abilities can be divided into first the direct incorporation of the specific affinity into the membranes bulk material, e.g. the specific adsorption of $\mathrm{Cu}^{2+}$ by wool keterose blended with silk fibron ${ }^{[13]}$ prior to electrospinning, second the post-modification of the nanofiber's surface, e.g. by grafting, ${ }^{[10]}$ and third by immobilizing MIP microspheres within the nanofiber membrane. ${ }^{[7-9]}$ We have chosen the latter approach since precipitation polymerization techniques were well established for the production of MIP microspheres and since electrospinning can be used for the production of membranes from a variety of materials such as natural and synthetic polymers ${ }^{[14]}$ providing the desired flexibility in tailoring the affinity membrane for the desired applications. Importantly, both precipitation polymerization and electrospinning can be operated at larger scale.

By electrospinning a solution of PAN and MIP microspheres, we obtained a composite PAN membrane with embedded MIP microspheres which was designed to provide specific affinity for cinchonidine. The membrane was flexible and mechanically sufficiently stable, to be handled manually. No membrane disintegration was observed during our affinity studies. The SEM image gives an insight into the microstructure of the membrane which consists of a network of PAN nanofibers as confirmed by Raman spectroscopy and embedded MIP microspheres which remained intact during the electrospinning process. The microspheres are not only adsorbed on the nanofibers' surface, but they are physically integrated into the nanofiber like a rope of pearls where the PAN nanofiber is the rope and the pearls are the microspheres. Whether the microspheres are still covered by a thin PAN film remains to be investigated. The nanofiber network is also mandatory for the membrane's high porosity. Macroscopically, the MIP 
microspheres were evenly distributed over the membrane's entire surface as was confirmed by D-ATR FT-IR spectroscopy at different positions of the membrane.

We aimed to study the influence of the PAN membrane matrix on the target binding affinity of the immobilized MIP microspheres in the composite PAN/MIP material, since the PAN nanofibers may alter the accessibility of the MIP microspheres for the target molecule. Yoshimatsu et al. ${ }^{[8]}$ have shown that their composite PET nanofiber MIP microspheres material remains still accessible for their target molecule propranolol, but they have not clarified at which extent the accessibility is influenced by the PET matrix. Our guest-binding experiments for the free MIP and NIP microspheres and for the immobilized ones within the PAN/MIP and PAN/NIP composite membranes show identical affinity for cinchonidine between the free and the immobilized microspheres and very low affinity for the PAN membrane without microspheres. We take this as evidence that the immobilization step does not hamper the intrinsic specific affinity of the MIP microspheres and suggest immobilization through electrospinning as a facile and effective tool for the immobilization of functional microspheres.

During our guest-binding experiment, $88 \%$ of the solution's cinchonidine was extracted by the PAN/MIP membrane. This corresponds to $0.5 \mathrm{mg}$ cinchonidine which were extracted by 100 $\mathrm{mg}$ composite material containing $20 \mathrm{mg}$ MIP microspheres, thus the composite membrane showed a target molecule capacity of 5 $\mathrm{mg} / \mathrm{g}$ membrane or $25 \mathrm{mg} / \mathrm{g}$ immobilized MIP microsphere at the given experimental conditions. This confirms the excellent binding affinity of the MIP microspheres for cinchonidine already mentioned by Lui et al.[6]

Recently, Bompart et al. ${ }^{[15]}$ reported the direct detection of the target binding to a moleculary imprinted polymer particle by confocal Raman spectroscopy on a surface-enhanced Raman spectroscopy (SERS) substrate using propranolol and atenolol as the target molecules. We were able to directly monitor the binding of our target molecule cinchonidine without the need for a SERS substrate. The Raman images provide also evidence that the target molecule cinchonidine only adsorbs onto the imprinted microspheres, while no specific adsorption is found to the PAN membrane. Some cinchonidine does accumulated at the intersection points of the PAN nanofibers. This is fully in line with our guest-binding experiments where only a small amount of cinchonidine was found to adsorb at the PAN membrane containing no MIP or NIP microspheres.

\section{Conclusion}

Molecularly imprinted polymer microspheres (MIPs) were immobilized in a PAN nanofiber membrane by electrospinning. The composite material shows the same affinity for the target molecule cinchonidine as do the free microspheres. The high affinity of the composite membrane for cinchonidine enabled us to localize the cinchonidine binding sites by Raman spectroscopy. Future work will focus on the long-term stability, workup, and reusability of the composite membrane, the microspheres to nanofibers size ratio, the optimal MIP loading in the composite material, and finally safety issues, i.e. leaching of the micro- and nanospheres.

Immobilization of functional microparticles in electrospun nanofiber membranes is a versatile and effective way to simplify the handling of micro- and nanoparticles (e.g. no centrifugation) while keeping their functionality which depends on their large surface area. The applied microspheres' synthesis and electrospinning techniques have the potential for scaling up and are very flexible in terms of the chosen polymer support and the microspheres' functionality: Therefore, we expect a broader application of composite nanofiber-microsphere membranes with specific recognition abilities as SPE or filtration material for de- contamination and purification purposes in water treatment as well as for food, pharmaceutical or fine chemical products.

\section{Acknowledgements}

The SEM image was acquired by the surface and nano analysis laboratory of Intertek Expert Services, P.O. Box 4002 Basel, Switzerland, Tel.: +41 68648 00. Partial funding from the start-up fond of the Department of Life Sciences and Facility Management of the ZHAW is gratefully acknowledged.

Received: January 26, 2011

[1] E. Smit, in 'Nanotechnology in Water Treatment Applications', Eds. T. E. Cloete, M. de Kwaadsteniet, M. Botes, Caister Academic Press, Norfolk, UK, 2010, pp 155-166.

[2] E. Lai, A. Dzhun, Z. de Maleki, in 'Trace Analysis with Nanomaterials", Eds. D. T. Pierce, J. X. Zhao, Wiley-VCH, Weinheim, Germany, 2010, pp 135-159.

[3] Y. Sueyoshi, C. Fukushima, M. Yoshikawa, J. Membrane Sci. 2010, 357, 90.

[4] M. Komiyama, T. Takeuchi, T. Mukawa, H. Asanuma, 'Molecular Imprinting: From Fundamentals to Applications', Wiley-VCH, Weinheim, Germany, 2003

[5] K. Yoshimatsu, K. Reimult, A. Krozer, K. Mosbach, K. Sode, L. Ye, Anal. Chim. Acta 2007, 584, 112

[6] Y. Liu, K. Hoshina, J. Haginaka, Talanta 2010, 80, 1713.

[7] I. S. Chronakis, A. Jakob, B. Hagström, L. Ye, Langmuir 2006, 22, 8960.

[8] K. Yoshimatsu, L. Ye, J. Lindberg, I. S. Chronakis, Biosens. Bioelectron. 2008, 23, 1208.

[9] K. Yoshimatsu, L. Ye, P. Stenlund, I. S. Chronakis, Chem. Commun. 2008, 2022.

[10] H. Zhang, H. Nie, D. Yu, C. Wu, Y. Zhang, C. J. B. White, L. Zhu, Desalination 2010, 256, 141.

[11] J. Matsui, N. Minamimura, K. Nishimoto, K. Tamaki, N. Sugimoto, J. Chromatogr. B 2004, 804, 223.

[12] J. Doshi, D. H. Reneker, J. Electrostatics 1995, 35, 151.

[13] C. S. Ki, E. H. Gang, I. C. Um, Y. H. Park, J. Membrane Sci. 2007, 302, 20.

[14] A. Greiner, J. H. Wendorff, Angew. Chem. Int. Ed. 2007, 46, 5670.

[15] M. Bompart, L. A. Gheber, Y. de Wilde, K. Haupt, Biosens. Bioelectron. 2009, 25,568 . 